\title{
The Implementation, in Matlab, of Three Digital Image Processing Algorithms to Evaluate the Change of Parameters of Two Consecutive Images
}

\author{
Virtyt Lesha \\ Polytechnic University of Tirana, Republic of Albania
}

\begin{abstract}
The assessment of quality of the image plays an important role in many processing applications developments. A great effort has been carried out in recent years in this field to develop and implement image quality metrics that correlate with measures of the quality expected. In fact, a great success this domain is not achieved. In this paper we have implemented three algorithms in Matlab that evaluate two consecutive images. These algorithms are: Mean Opinion Score (MOS), Peak Signal to Noise Ratio (PSNR) and Structural Similarity Index (SSIM).Currently, we have considered 50 pairs of consecutive images retrieved from a video with a difference of $1 \mathrm{~ms}$, which will be subject of entry in these three algorithms developed in Matlab. After we enter these couples of images, three numbers will appear which define the level of changing the two images parameters. The results generated will be given through statistical conclusions of spline interpolation fitting. Finally, the limitations of this study concentrate on the development and improvement of these three algorithms in such a way that the evaluation must be done as correctly as possible in cases where the difference in pixels between the two images is larger.
\end{abstract}

Keywords: MOS, PSNR, SSIM, development, Matlab, spline JEL classification: C00, C40

\section{Introduction}

The image processing consists in the use of mathematical operations using any form of signal processing for which the entry is an image, such as a photo, video frame, etc; the output of image processing can be an image or a set of characteristics or parameters related to the image. Techniques used include treating the image processing as a two-dimensional signal and applying signal processing techniques to the images. Usually, image processing refers to digital image processing; however in today's technologies the optical digital and analogue processing is possible. This article discusses techniques that are applied to images.

A field of study that is closely related to the processing of images are the computer graphics and computer vision. In computer graphics, images are manually performed by physical models of objects and premises. Computer vision, on the other hand, is often considered as high level processing of images, where a computer/software aims to decode the physical content in an image or a sequence of images (for example, scans Magnetic 3D).

In modern science and technology, the images also have a greater vision regarding expansion due to ever increasing importance of scientific visualisation (or often given data of the large and complex escalation in the scientific/experimental 
aspect). Examples of these elements are the microwave research, genetic research, etc.

Mean opinion score (MOS) is a test that has been used for decades in the telephone network to take advantage of network quality. In multimedia MOS (audio, image, videol is used especially when we present codec-s to compress the requirements of bandwidth (for example, a link voice or image digitalized by a PCM $64 \mathrm{kilobit} / \mathrm{sec}$ modulation). MOS provides an indicator of numeric which shows the quality of the environment perspective to take after the compression and/or transmission. MOS is expressed as an integer in the range 1 to 5,1 being the lowest quality, and 5 is the biggest quality received. (Ferrara et al., 1992)

The structural similarity index (SSIM) is a method of predicting the quality of digital video and digital imaging. SSIM used to measure the similarity between two images. SSIM index is a full reference metrics; in other words the measurement or prediction of the quality of the image is based on an image of the original uncompressed as a reference. SSIM is designed to improve traditional methods such as PSNR (peak signal-to-noise ratio) and MSE (Mean square error).

Peak signal-to-noise ratio (PSNR) is an engineering term that expresses the maximum power level possible tucked to distorting the signal and noise power affecting the credibility of the presentation of a signal such as the image. Because many signals have a very wide range, PSNR is usually expressed in logarithmic scale decibels (dB).(Bruce et al., 2001)PSNR is the classic method used to measure the quality of reconstruction of the images. The signal in this case is the original data and the noise is introduced in the compression error. When comparing compression codec-s, generally PSNR is an approximation to the human perception of quality reconstruction. Although a large PSNR value generally indicates that reconstruction is a high quality, in some cases it may not be such. Actually, we should be very careful with the range of validity of this metric; he is definitely valid when used to compare results from the same codec and the same content.

The aim of the paper is to give a trend-line, in terms of spline-cubic approximation, of the performance of these three algorithms.

In the section of the methodology we have treated the scheme of the simulations which consists in using 50 couples of video frames and entering them in the three algorithms.

The next section treats the results of these simulations. These results consist in calculating the spline cubic curve equation as well as the respective coefficient of determination.

Finally, in the last section we have treated the limitations and suggestions of the paper and the approach in question

\section{Methodology and data}

In this paper we have implemented three image-processing algorithms: mean opinion score, structural similarity index and peak signal-to-noise ratio.

Initially, we have generated 50 couples of video frames with time intervals of $1 \mathrm{~ms}$ difference. This process is accomplished through the software "Windows Movie Maker". These frames consist of images that the eye of man does not have any significant difference, although not excluded the case where the human eye can distinguish clearly the differences between consecutive images. This fact is evident in the figure below. 


\section{Figure 1}

The figure showing two consecutive pictures for which the human eye can notice the differences between them

Source: www.elance.com (freelancer website)

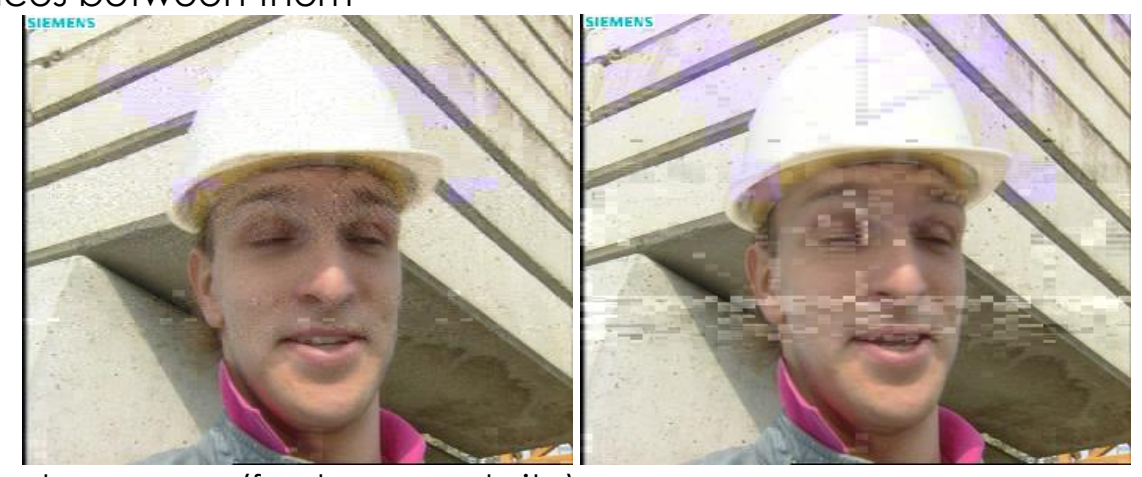

After generating these 50 pairs of images, the latter will be as an input through 3 algorithms: MOS, SSIM and PSNR. The output of these three algorithms will consist of three numbers respectively indicating the level of difference between the two images. So, we will gain a database of 150 values that 50 of them come from MOS, 50 of SSIM and 50 of PSNR.

These data or in other words, these numbers will be addressed through statistical methods to observe the trend-line of these values. The method used to determine this trend-line is spline interpolation fitting implemented in Matlab. We will define every number generated from the algorithms an ID number and this ID will be used as abscissa of the three graphs.

Finally, we will present the equations of the respective spline-cubic curves with the respective coefficient of determination.

\section{The results of the simulations}

In the mathematical field of numerical analysis, a spline interpolation is a form of interpolation where the interpolant is a special type of a polynomial called spline. Spline interpolation often treated preferably by polynomial interpolation because the interpolation error can be smaller when the polynomials use lower orders for the spline. Spline interpolation avoids the problem of Runge's phenomenon, in which the fluctuations can occur between points when using polynomial interpolation with higher orders. In this paper we have implemented the spline cubic polynomial.(Boashash, et al., 1992, 1987)

We have presented in the following figures the dependency graphs of the couples of consecutive images with $1 \mathrm{~ms}$ interval and curve the spline cubic lines together with the relevant equation.

Let's look at the functional connectivity of the dependence results of the MOS implementation of the 50 sample couples. 
Figure 2

The dependency of MOS results of the couples of images as well as the curve of the spline cubic curve

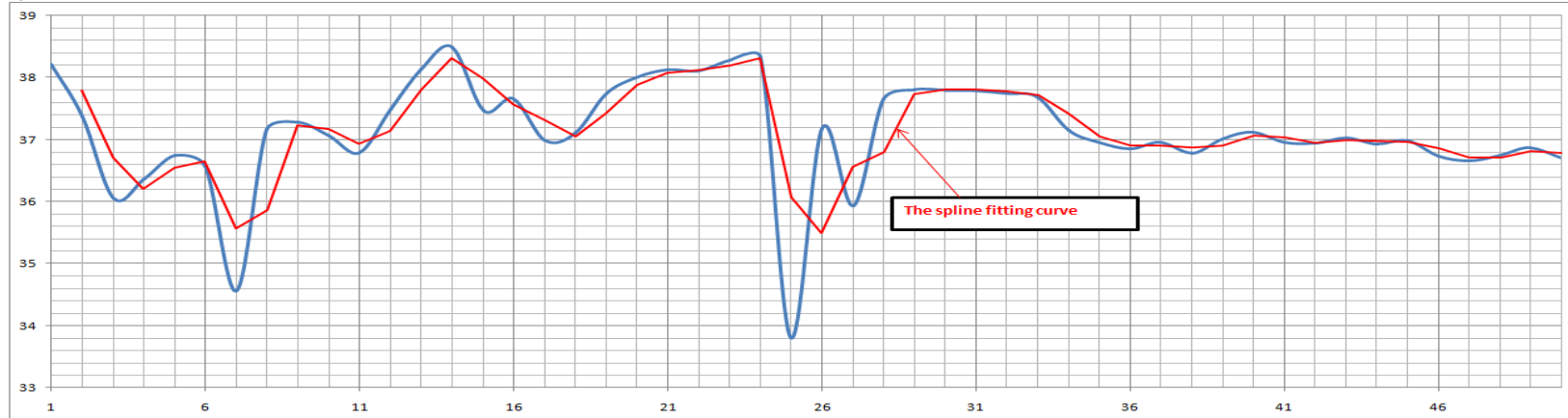

Source: Matlab simulation results

The blue line expresses the dependence of the MOS implementations and the red line expresses the spline cubic curve.

The equation of the spline cubic is:

$$
y=34 * x^{3}+5.34 * x^{2}+34 * x+56.32
$$

The coefficient of determination is $R^{2}=0.931$. The rest of dependence remains to be studied.

Let's look at the functional connectivity of the dependence results of the SSIM implementation of the 50 sample couples.

Figure 3

The dependency of SSIM results of the couples of images as well as the curve of the spline cubic curve

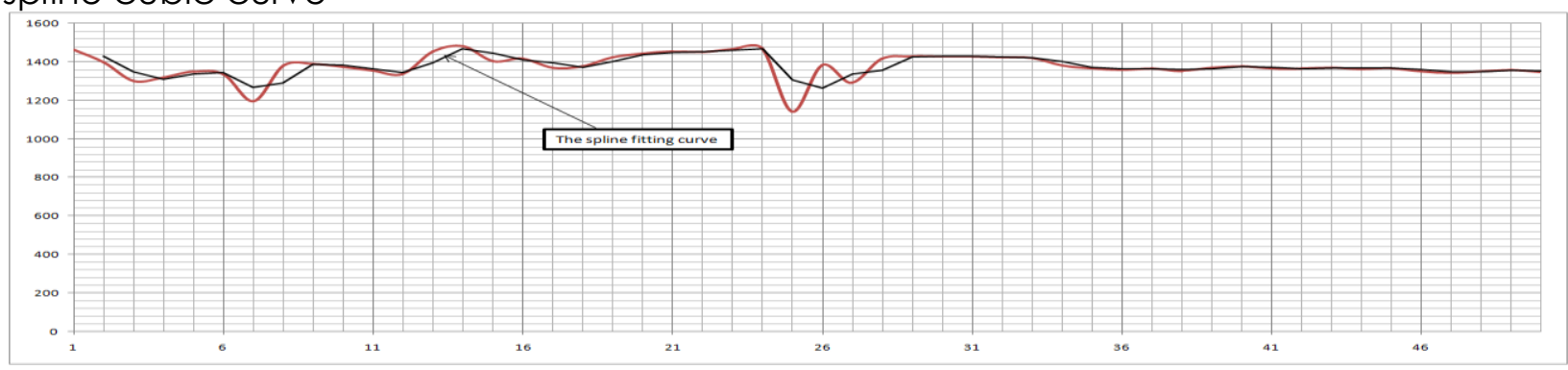

Source: Matlab simulation results

The red line expresses the dependence of the SSIM implementations and the black line expresses the spline cubic curve.

The equation of the spline cubic is:

$$
y=45 * x^{3}+7.89 * x^{2}+12 * x+78.43
$$

The coefficient of determination is $R^{2}=0.871$. The rest of dependence remains to be studied.

Let's look at the functional connectivity of the dependence results of the PSNR implementation of the 50 sample couples. 
Figure 4

The dependency of PSNR results of the couples of images as well as the curve of the spline cubic curve

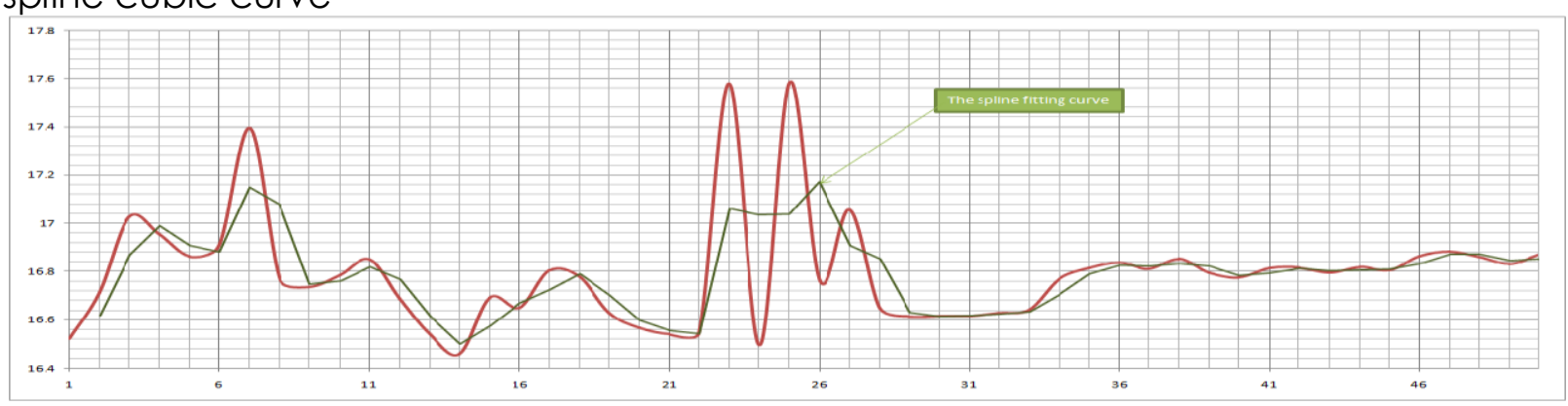

Source: Matlab simulation results

The red line expresses the dependence of the PSNR implementations and the black line expresses the spline cubic curve.

The equation of the spline cubic is:

$$
y=98^{*} x^{3}+7.43^{*} x^{2}+56^{*} x+1.93
$$

The coefficient of determination is $R^{2}=0.235$. The rest of dependence remains to be studied.

\section{Conclusions}

In this article we gave the results of the simulation via Matlab (of the dependency of the tendency of the spline cubic interpolation of the difference of the image parameters of two consecutive ones. The algorithms used and implemented in Matlab were Mean Opinion Score (MOS), Peak Signal to Noise Ratio (PSNR) and Structural Similarity Index (SSIM) (Akansu et al., 1992).

The first results of this paper consisted in presenting the spline cubic dependency of the difference of the consecutive MOS algorithm. (Cohen et al., 1989)

The second results of this paper consisted in presenting the spline cubic dependency of the difference of the consecutive SSIM algorithm and the last results of this paper consisted in presenting the spline cubic dependency of the difference of the consecutive PSNR algorithm (Cichicki, 2002).

Finally I propose as future work the use of another approach of interpolation methods that can be more effective to define the trend of these data (Aldroubi et al., 1996). Also, I propose that the standard MOS, SSIM and PSNR algorithm be improved in such a way to minimize the time needed to find the difference between the two images as well as to find a hybrid algorithm such as can applied in video files.

The limitations of the study consist in representing results of such structures of algorithms that perform with greater speed in the terms of time. If, the execution time of these algorithms should be smaller, the better the performance should be better. The issues of this study are treated at the studies of (Bruce, 2001) where a lot of variations of digital image processing algorithms have been treated.

\section{References}

1. Akansu, A.N., Haddad, R.A. (1992), "Multiresolution Signal Decomposition: Transforms, sub bands, wavelets". Academic Press, San Diego CA. A modern classic that presents, among other things, some of the underlying theoretical aspects of wavelet analysis. 
2. Aldroubi A., Unser, M. (1996), "Wavelets in Medicine and Biology", CRC Press, Boca Raton, FL. Presents a variety of applications of wavelet analysis to biomedical engineering.

3. Boashash, B. (1992), "Time-Frequency Signal Analysis", Longman Cheshire Pty Ltd. Early chapters provide a very useful introduction to time-frequency analysis followed by a number of medical applications.

4. Boashash, B., Black, P.J. (1987), "An efficient real-time implementation of the WignerVille Distribution", IEEE Trans. Acoust. Speech Sig. Proc. ASSP-35:1611-1618. Practical information on calculating the Wigner-Ville distribution.

5. Bruce, E. N. (2001),"Biomedical Signal Processing and Signal Modelling", John Wiley and Sons, New York. Rigorous treatment with more of an emphasis on linear systems than signal processing. Introduces nonlinear concepts such as chaos.

6. Cichicki, A., Amari S. (2002), "Adaptive Blind Signal and Image Processing: Learning Algorithms and Applications", John Wiley and Sons, Inc. New York. Rigorous, somewhat dense, treatment of a wide range of principal component and independent component approaches. Includes disk.

7. Cohen, L. (1989),"Time-frequency distributions - A review". Proc. IEEE 77:941-981. Classic review article on the various time-frequency methods in Cohen's class of timefrequency distributions.

8. Elance. Freelancer Website. Main page. Available at:www.elance.com (15/5/2016)

9. Ferrara, E., Widrow, B. (1992), "Fetal Electrocardiogram enhancement by timesequenced adaptive filtering". IEEE Trans. Biomed. Engr. BME-29:458-459. Early application of adaptive noise cancellation to a biomedical engineering problem by one of the founders of the field.

\section{About the author}

Virtyt Lesha is electronics engineer specialised in digital signal and image processing and automatic control systems through Matlab. Part of his experience is the scientific research that consists in the fact that of publishing 3 books in Germany, 8 scientific papers in international conferences in Albania, Montenegro, Croatia, Slovak Republic, Bulgaria, 6 scientific papers in international journals in USA, Germany, United Kingdom, 1 technical report in India and finally 4 abstracts/presentations in Croatia and Albania. The author can be contacted at virtytl@hotmail.com. 